\title{
Modelos de estimativa da irradiação solar global em superfície vertical
}

\author{
Estimation models of global solar irradiation in vertical surface \\ Modelos de estimación de la irradiación solar global en superficie vertical
}

Recebido: 02/06/2021 | Revisado: 08/06/2021 | Aceito: 11/06/2021 | Publicado: 26/06/2021

\author{
Camila Piacitelli Tieghi \\ ORCID: https://orcid.org/0000-0001-8060-5373 \\ Universidade Estadual Paulista, Brasil \\ E-mail: camila.piacitelli@unesp.br \\ Alexandre Dal Pai \\ ORCID: https://orcid.org/0000-0002-1283-901X \\ Universidade Estadual Paulista, Brasil \\ E-mail: dal.pai@unesp.br
}

\begin{abstract}
Resumo
A radiação solar é uma fonte energética vital de todos processos biológicos, químicos, físicos e atmosféricos. Além disso, atualmente, com uma preocupação crescente no que se refere autilização de fontes alternativas e sustentáveis de energia, seu uso tem sido utilizado como uma boa solução para conversão energética, controle de temperatura de água e conforto térmico. Para cada local onde será feita a utilização da radiação solar, é preciso conhecer a variação anual dos níveis quantitativos energéticos incidentes, pois em cada região do planeta, a incidência da radiação solar global apresenta diferentes variações anuais. Quando se trabalha em uma superfície inclinada, a orientação e o ângulo da inclinação da superfície alteram os níveis energéticos recebidos se comparados com uma superfície horizontal. Medições da irradiação solar em plano inclinado não são corriqueiras em estações meteorológicas devido à entraves financeiros, de instrumentação e de operação. Por este motivo, o potencial energético e térmico da irradiação solar em superfície vertical é deixado de lado. Este trabalho teve como objetivo avaliar os dados da irradiação solar global em superfície vertical orientada ao Norte medidos durante o ano de 2010 na estação de radiometria do Departamento de Engenharia Rural da Faculdade de Ciências Agronômicas da UNESP de Botucatu. Foram testados modelos clássicos da literatura para estimatimar da irradiação global desenvolvidos em superfícies inclinadas e foram comparados com dados da estação de radiometria de Botucatu-SP medidos em superfície vertical. No final, foi proposto um modelo de estimativa da irradiação solar global para superfície vertical para Botucatu-SP.
\end{abstract}

Palavras-chave: Inclinada; Radiação solar; Modelagem; Vertical.

\begin{abstract}
Solar radiation is a vital energy source for all biological, chemical, physical and atmospheric processes. In addition, nowadays, with a growing concern in relation to the use of alternative and sustainable sources of energy, its use has been used as a good solution for energy conversion, water temperature control and thermal comfort. For each place where the use of solar radiation will be made, it is necessary to know the annual variation of the quantitative energy levels incident, because in each region of the planet, the behavior of the incident global solar radiation presents different annual variations. When working on a sloping surface, the orientation and angle of the slope of the surface changes the energy levels received when compared to a horizontal surface. Measures of solar radiation on an inclined plane are not common in meteorological stations due to financial, instrumentation and operating barriers. For this reason, the energetic and thermal potential of solar radiation on a vertical surface is neglected. This work aimed to evaluate the global solar irradiation data on a vertical surface oriented to the North measured during 2010 at the radiometry station of the Rural Engineering Department of the Faculty of Agronomic Sciences at UNESP in Botucatu. Classical literature models were tested to estimate global irradiation developed on inclined surfaces and were compared with data from the Botucatu-SP radiometry station measured on a vertical surface. In the end, a model for estimating the global solar irradiation for vertical surface was proposed for Botucatu-SP.
\end{abstract}

Keywords: Inclined; Solar radiation; Modeling; Vertical.

\section{Resumen}

La radiación solar es una fuente de energía vital para todos los procesos biológicos, químicos, físicos y atmosféricos. Además, en la actualidad, con una preocupación creciente en relación al uso de fuentes de energía alternativas y sostenibles, su uso se ha utilizado como una buena solución para la conversión de energía, control de temperatura del agua y confort térmico. Para cada lugar donde se hará el uso de la radiación solar, es necesario conocer la variación anual de los niveles cuantitativos de energía incidente, pues en cada región del planeta, el comportamiento de la radiación solar global incidente presenta diferentes variaciones anuales. Cuando se trabaja en una superficie inclinada, la orientación y el ángulo de la pendiente de la superficie cambian los niveles de energía recibidos en comparación con 
una superficie horizontal. Las medidas de radiación solar en un plano inclinado no son comunes en las estaciones meteorológicas debido a barreras financieras, de instrumentación y operativas. Por este motivo, se desprecia el potencial energético y térmico de la radiación solar sobre una superficie vertical. Este trabajo tuvo como objetivo evaluar los datos de irradiación solar global sobre una superficie vertical orientada al Norte medidos durante 2010 en la estación de radiometría del Departamento de Ingeniería Rural de la Facultad de Ciencias Agronómicas de la UNESP en Botucatu. Se probaron modelos de literatura clásica para estimar la irradiación global desarrollada en superficies inclinadas y se compararon con datos de la estación de radiometría Botucatu-SP medidos en una superficie vertical. Al final, se propuso un modelo para estimar la irradiación solar global para superficie vertical para Botucatu-SP.

Palabras clave: Inclinada; Radiación solar; Modelado; Vertical.

\section{Introdução}

A ciência da irradiação solar em plano vertical é importante em diversas áreas, visando o conforto térmico ou para utilização da energia solar para conversão energética ou térmica. Na zootecnia, a informação da incidência solar nas fachadas das construções de galpões avícolas é necessária para aumentar o conforto térmico dos animais (Carvalho, et al., 2014; Sevegnani, Ghelfi Filho \& Silva, 1994). Na arquitetura, os dados de irradiação solar são importantes para projetar edifícios com eficiência energética, onde eles são normalmente usados em aplicações como o cálculo de ganhos de calor solar ou cargas de resfriamento. Os níveis de irradiação solar nas fachadas influem na forma da edificação, orientação das aberturas, dimensão e posicionamento das paredes, proteções solares nas aberturas e materiais utilizados em paredes (Subhashini \& Thirumaran, 2018; Yao et al., 2018). Na agronomia, o conhecimento dos níveis de irradiação solar incidentes nas fachadas de estufas solares são de suma importância para equilibrar a temperatura interna nos meses de verão e inverno e visando também a economia de uso do combustível fóssil ou de qualquer outro sistema de aquecimento usado para aquecer a estufa no período do inverno (ElMaghlany, Teamah \& Tanaka, 2015; Stanciu, Stanciu \& Dobrovicescu, 2016). Na engenharia elétrica, o potencial solar das fachadas dos prédios é estudado para fixar painéis fotovoltaicos, pois as fachadas contribuem significativamente para o potencial solar total dos prédios (Brito, et al., 2017; Redweik; Redweik, Catita \& Brito, 2013).

A irradiação solar global em superfície vertical é composta de três porções. A porção direta, originário do disco solar quando este está total ou parcialmente visível. A porção difusa, produzida a partir da ação de espalhamento causado na atmosfera local, sofrendo um ou mais desvios até incidir sobre um local. E por último, a porção refletida, proveniente da reflexão dos raios solares incidentes sobre albedo da superfície. O albedo varia de acordo com seus componentes físicos (cor, rugosidade, etc.) e do ângulo zenital (Varejão-Silva, 2006).

Estações meteorológicas comumente medem a irradiação solar global em superfícies horizontais. No entanto, a medida da irradiação global em superfície vertical é escassa devido à entraves financeiros, de instrumentação e de operação.

Para conhecer os níveis de irradiação solar global em plano inclinado em determinado local, quando não é possível obter suas medidas, os modelos de estimativa da irradiação solar auxiliam nessa tarefa. Vários autores propuseram modelos que estimam a irradiação solar em plano inclinado com base nas medidas da irradiação solar na horizontal, por ser um dado medido em estações meteorológicas corriqueiramente (Hay, 1979; Klucher, 1979; Liu \& Jordan, 1963; Perez, et al., 1987; Temps \& Coulson, 1977).

Existem três tipos de modelagem de estimativa da irradiação solar. Os modelos paramétricos, de redes neurais e os estatísticos. Os modelos paramétricos necessitam de várias informações das condições atmosféricas locais dependendo da componente da radiação solar a ser estimada ou então a utilização de percentuais fixos de ocorrência de cada componente da radiação solar global (Yıldırım, Teke \& Antonanzas-Torres, 2018). Com a modelagem realizada através de rede neural artificial (RNA) é possível realizar as previsões e avaliações a partir de dados meteorológicos disponíveis, tais como a temperatura ambiente, irradiação solar, umidade do solo, sensação térmica, umidade do ar, orvalho, precipitação (Barth, et al., 2016). Por fim, os modelos estatísticos necessitam de dados medidos para serem confrontados com os valores estimados. Os modelos estatísticos são equações matemáticas ajustadas por meio de regressão linear ou polinomial em correlações com as frações 
radiométricas ou com a razão de insolação (Buriol, et al., 2012). Dentre tantos modelos de estimativa da irradiação solar, poucos foram testados afim de estimar a irradiação solar em plano vertical.

Os modelos da literatura que foram testados a fim de estimar a irradiação solar em plano inclinado são para superfícies inclinadas para o Equador. No hemisfério Sul, orientado ao Norte e no hemisfério Norte, orientado ao Sul. Isto se dá pelo fato de que quando se inclina uma superfície para o Equador, simula-se uma superfície horizontal em latitude maior, no caso de locais no hemisfério Sul, visando maximizar o ganho energético da irradiação solar, principalmente nos períodos de inverno. Devido à declinação solar, nos períodos de inverno para o hemisfério Sul, o Sol ilumina a região Norte do planeta.

Para aumentar a precisão da estimativa da irradiação solar, pode-se combinar modelos de séries temporais e espaciais. Enquanto os modelos de séries temporais estimam a irradiação solar apenas para um local, os modelos espaciais podem estimar a irradiação solar para muitos locais. Um complementa o outro, pois, para modelos de séries temporais se tem muitos anos de dados enquanto para modelagem espacial não se tem muitos anos de dados.

Modelos de séries temporais são conjuntos de observações ordenadas no tempo, que podem ser contínuas, quando observações são feitas continuamente no tempo, ou discretas, quando as medidas são tomadas em intervalos de tempo regularmente espaçados (Morettin \& Toloi, 2004). Modelos espaciais, que são modelos de estimativa por satélites geoestacionários, são uma importante fonte de informação, dadas a frequência elevada, a resolução espacial das estimativas, e o número de anos atualmente disponível. São simples de manipular, e ainda permitem resgatar detalhes para estudos climatológicos do padrão comportamental da radiação solar (Ceballos \& Bottino, 2007).

O trabalho tem os seguintes objetivos:

a) Testar modelos clássicos da literatura para a estimativa da irradiação global desenvolvidos em superfícies inclinadas com dados da estação de radiometria de Botucatu-SP medidos em superfície vertical.

b) Propor um modelo de estimativa da irradiação solar global para superfície vertical para Botucatu-SP.

\section{Metodologia}

\subsection{Local e clima}

O estudo é baseado numa pesquisa quantitativa das medidas da irradiação global em planos horizontal e vertical orientado ao Norte geográfico estação radiométrica do Departamento de Engenharia Rural da Faculdade de Ciências Agronômicas da UNESP de Botucatu (latitude 22 $54^{\prime} \mathrm{S}$, longitude $48^{\circ} 27^{\prime} \mathrm{O}$ e altitude $716 \mathrm{~m}$ ). Foram utilizados os dados medidos no ano de 2010. A cidade de Botucatu tem cerca de 130.000 habitantes e é rodeada por uma formação de relevo assimétrico denominada Cuesta de Botucatu e pelas bacias hidrográficas do Tietê e do Paranapanema. De acordo com a classificação climática de Köppen, o clima local é o Cwa (clima subtropical úmido), com verão quente, úmido e chuvoso e inverno seco e de temperaturas amenas. Os dados de temperatura e umidade relativa seguem as variações astronômicas, com valores máximos de temperatura e umidade relativa em fevereiro $\left(23,12{ }^{\circ} \mathrm{C}\right.$ e $78,25 \%$ respectivamente) e valores mínimos de temperatura em julho $\left(17,10^{\circ} \mathrm{C}\right)$ e umidade relativa em agosto $(63,97 \%)$. A estação chuvosa ocorre no verão e primavera, acompanhada de elevada nebulosidade. Cerca de $80 \%$ da precipitação anual ocorre nesse período, com máxima no mês de janeiro (246,2 mm). Nas estações do inverno e outono, a precipitação média é inferior a 100 mm mensais, com mínima em agosto (36,1 mm) (Codato, et al., 2007).

\subsection{Instrumentação e controle de qualidade}

Os valores das irradiâncias solares foram monitorados por um sistema de aquisição de dados automático modelo Datalogger 23X da empresa Campbell Scientific Inc com frequência de varredura de 0,2Hz, armazenando médias de 5 minutos no formato $\mathrm{W} / \mathrm{m}^{2}$, de janeiro à dezembro de 2010. 
A irradiância solar global horizontal foi medida com piranômetro Eppley-PSP. A irradiância solar global no plano vertical foi medida com piranômetro Kipp-Zonen - CM3. Para medir a irradiância global na superfície vertical, o piranômetro foi posicionado sobre plano inclinado à $90^{\circ} \mathrm{com}$ a face orientada para o Norte.

Foi realizada uma filtragem dos dados, dos quais foram utilizados no total 338 dias, que equivalem a $92,6 \%$ do total de um ano. As irradiâncias global $\left(H_{g}^{d}\right)$, direta $\left(H_{b h}^{d}\right)$ e difusa $\left(H_{d}^{d}\right)$ em superfície horizontal e irradiância global em superfície vertical foram integradas em partições diárias de tempo, do nascer ao pôr do Sol, em $\mathrm{MJ} / \mathrm{m}^{2}$. A irradiação solar extraterrestre em superfície horizontal $\left(H_{0}^{d}\right)$ foi calculada segundo Iqbal (1983) (Iqbal, 1983).

\subsection{Modelagem}

A irradiação solar global diária $\left(H_{\beta T}^{d}\right)$ incidente em determinada superfície inclinada a um ângulo $\beta$ em relação ao plano horizontal é estimada pela soma das três frações da irradiação solar global, irradiação difusa $\left(H_{\beta d}^{d}\right)$, que são a irradiação direta $\left(H_{\beta b}^{d}\right)$ e a irradiação refletida $\left(H_{\beta r}^{d}\right)$ conforme equação 1.:

$$
H_{\beta T}^{d}=H_{\beta b}^{d}+H_{\beta d}^{d}+H_{\beta r}^{d}
$$

Os modelos de estimativa testados no trabalho foram: modelo Geométrico (Iqbal, 1983), Liu \& Jordan (1963), Temps e Coulson (1977), Perez (1987), Klein (1976) e Hay (1979), apresentando diferenças em suas formulações em função de parâmetros atmosféricos e condições atmosféricas distintas. Todos esses modelos foram parametrizados para estimar a porção difusa, sendo as porções direta e refletida as mesmas para todos os modelos.

A partição da irradiação direta $\left(H_{\beta b}^{d}\right)$ incidente em superfícies inclinadas (Iqbal, 1983) é calculada corrigindo a irradiação direta projetada na horizontal $\left(H_{b h}^{d}\right)$, por meio do fator de correção geométrico $R_{B}$, conforme a Equação 2:

$$
H_{\beta b}^{d}=H_{b h}^{d} R_{B}
$$

A partição da irradiação solar refletida $\left(H_{\beta r}^{d}\right)$ incidente em uma superfície inclinada, considerando que a reflexão da superfície como sendo isotrópica, é calculada pela Equação 3, a seguir:

$$
H_{\beta r}^{d}=H_{g}^{d} \rho \frac{1}{2}(1-\cos (\beta))
$$

Onde, $\rho$ é o albedo, determinado como a razão entre a quantidade de luz que é difundida ou refletida por uma superfície e a quantidade de luz incidente sobre a mesma, $\beta$ é o ângulo de inclinação da superfície, $H_{g}^{d}$ é a irradiação global horizontal e o termo $\left[\frac{1}{2}(1-\cos (\beta)]\right.$ é a divisão entre a irradiação solar incidente na superfície inclinada sobre a irradiação solar refletida pelo solo.

Já para a fração difusa $\left(H_{\beta d}^{d}\right)$, a Tabela 1 traz as equações para casa um dos modelos de estimativa testados. 
Research, Society and Development, v. 10, n. 7, e38910716721, 2021

(CC BY 4.0) | ISSN 2525-3409 | DOI: http://dx.doi.org/10.33448/rsd-v10i7.16721

Tabela 1 - Modelos de estimativa da irradiação solar difusa em superfície inclinadas

\begin{tabular}{|c|c|c|c|}
\hline Autores & Local & Modelo & Isotropia \\
\hline Geométrico & & $H_{\beta d}^{d}=H_{d}^{d} R_{B}$ & Não considera \\
\hline $\begin{array}{l}\text { Liu \& Jordan } \\
\text { (1963) }\end{array}$ & $\begin{array}{l}\text { Massachusetts } \\
\qquad(\text { EUA) }\end{array}$ & $H_{\beta d}^{d}=H_{d}^{d} \frac{1}{2}(1+\cos (\beta))$ & Isotrópico \\
\hline Temps \& & Califórnia (EUA) & $H_{\beta d}^{d}=H_{d}^{d}(1+\cos (\beta))\left[1+\operatorname{sen}^{3}\left(\frac{\beta}{2}\right)\right][1$ & Anisotrópico \\
\hline Coulson (1977) & & $\left.+\cos ^{2}\left(\theta_{\beta}\right) \operatorname{sen}^{3}\left(\theta_{Z}\right)\right]$ & \\
\hline Hay (1979) & $\begin{array}{l}\text { Vancouver } \\
\text { (Canada) }\end{array}$ & $\begin{aligned} H_{\beta d}^{d}=I_{c}+I_{S I}= & H_{d}^{d}\left[A_{I} R_{B}+\frac{1}{2}(1\right. \\
& \left.+\cos (\beta))\left(1-A_{I}\right)\right]\end{aligned}$ & Anisotrópico \\
\hline Klucher (1979) & Cleveland (EUA) & $H_{\beta d}^{d}=H_{d}^{d}(1+\cos (\beta))\left[1+\operatorname{sen}^{3}\left(\frac{\beta}{2}\right)\right][1$ & Anisotrópico \\
\hline & & $\left.+F \cos ^{2}\left(\theta_{\beta}\right) \operatorname{sen}^{3}\left(\theta_{Z}\right)\right]$ & \\
\hline Perez (1987) & Albany, NY (EUA) & $\begin{aligned} H_{\beta d}^{d}=H_{d}^{d}\left[\frac{1}{2}(1+\right. & \cos (\beta))\left(1-F_{1}\right)+F_{1} R_{B} \\
& \left.+F_{2} \operatorname{sen}(\beta)\right]\end{aligned}$ & Anisotrópico \\
\hline
\end{tabular}

Símbolos:

$H_{\beta d}^{d}$ : Irradiação solar difusa em plano inclinado $\left(\mathrm{MJ} / \mathrm{m}^{2}\right)$

$H_{d}^{d}$ : Irradiação difusa incidente na superfície horizontal $\left(\mathrm{MJ} / \mathrm{m}^{2}\right)$

$R_{B}$ : Fator de correção da incidência dos raios solares

$\beta$ : Ângulo de inclinação da superfície (graus)

$\theta_{\beta}$ : Ângulo de incidência (graus)

$\theta_{Z}$ : Ângulo Zenital (graus)

$I_{c}$ : Irradiação difusa circunsolar $\left(\mathrm{MJ} / \mathrm{m}^{2}\right)$

$I_{S I}$ : Irradiação difusa isotrópica $\left(\mathrm{MJ} / \mathrm{m}^{2}\right)$

$A_{I}$ : Índice anisotrópico $A_{I}=\left(\frac{H_{b h}^{d}}{H_{0}^{d}}\right)$

$F$ : Função modeladola $\left[F=1-\left(H_{d}^{d} / H_{g}^{d}\right)^{2}\right]$

$F_{1}$ : Irradiação difusa circunsolar $\left(\mathrm{MJ} / \mathrm{m}^{2}\right) F_{1}=F_{11}+F_{12} \Delta+F_{13} \theta_{Z}$

$F_{2}$ : Irradiação difusa horizontal $\left(\mathrm{MJ} / \mathrm{m}^{2}\right) F_{2}=F_{21}+F_{22} \Delta+F_{23} \theta_{Z}$

Fonte: Autores (2021).

No modelo de Perez, et al. (1986) é necessário realizar cálculos para se obter valores que serão utilizados nas equações da irradiação difusa circunsolar $\left(F_{1}\right)$ e irradiação difusa horizontal $\left(F_{2}\right)$, os quais estão apresentados nas equações 4 , 5 e 6 e

Tabelas 2 e 3, a seguir:

$$
\begin{gathered}
\Delta=m_{r} H_{d}^{d} / H_{0}^{d} \\
m_{r}=\frac{1}{\left[\cos \theta_{Z}+0,15\left(93,885-\theta_{Z}\right)\right]^{-1,253}} \\
e=\left(H_{d}^{d}+H_{b}^{d}\right) / H_{d}^{d}
\end{gathered}
$$

Onde: $H_{b}^{d}$ : Irradiação direta horizontal; $H_{0}^{d}$ : Irradiação solar extraterrestre em superfície horizontal; $\Delta$ : Índice atmosférico; $m_{r}$ : Massa ótica do ar; $e$ : Índice de turbidez de céu; $\theta_{Z}$ Ângulo zenital (em radianos). 
Research, Society and Development, v. 10, n. 7, e38910716721, 2021

(CC BY 4.0) | ISSN 2525-3409 | DOI: http://dx.doi.org/10.33448/rsd-v10i7.16721

Tabela 2 - Intervalos de $\boldsymbol{e}$ para cada categoria de turbidez de céu

\begin{tabular}{cr}
\hline Categorias de $e$ & Intervalos de $e$ \\
\hline 1 & $1,056<e \leq 1,056$ \\
2 & $1,253<e \leq 1,586$ \\
3 & $1,586<e \leq 2,134$ \\
4 & $2,134<e \leq 3,230$ \\
5 & $3,230<e \leq 5,980$ \\
6 & $5,980<e \leq 10,080$ \\
7 & $>10,080$ \\
\hline
\end{tabular}

Fonte: Perez, et al. (1987).

Tabela 3 - Coeficientes $\boldsymbol{F}_{\mathbf{1}}$ e $\boldsymbol{F}_{\mathbf{2}}$ para cada categoria de $\boldsymbol{e}$, compilados de Perez et al. (1987)

\begin{tabular}{ccccccc}
\hline Categorias de $e$ & $F_{11}$ & $F_{12}$ & $F_{13}$ & $F_{21}$ & $F_{22}$ & $F_{23}$ \\
\hline 1 & 0,041 & 0,621 & $-0,105$ & $-0,004$ & 0,074 & $-0,031$ \\
2 & 0,054 & 0,966 & -0166 & $-0,016$ & 0,114 & $-0,045$ \\
3 & 0,227 & 0,866 & $-0,250$ & 0,069 & $-0,002$ & $-0,062$ \\
4 & 0,486 & 0,670 & $-0,373$ & 0,148 & $-0,137$ & $-0,056$ \\
5 & 0,819 & 0,106 & $-0,465$ & 0,268 & $-0,497$ & $-0,029$ \\
6 & 1,020 & $-0,260$ & $-0,514$ & 0,306 & $-0,804$ & 0,046 \\
7 & 1,009 & $-0,708$ & $-0,433$ & 0,287 & $-1,286$ & 0,166 \\
8 & 0,936 & $-1,121$ & $-0,352$ & 0,226 & $-2,449$ & 0,383 \\
\hline
\end{tabular}

Fonte: Perez, et al. (1987).

\subsection{Indicativos estatísticos}

A performance dos modelos de estimativa da irradiação solar em plano vertical foi verificado por meio dos indicativos estatísticos MBE (Mean Bias Error), RMSE (Root Mean Square Error) (Stone, 1993).

$$
\begin{gathered}
\operatorname{MBE}=\left(\frac{\sum_{i}^{N}\left(y_{i}-x_{i}\right)}{N}\right) \\
\operatorname{MBE}(\%)=100 \frac{\left(\sum_{i}^{N}\left(y_{i}-x_{i}\right) / N\right)}{\bar{x}} \\
\operatorname{RMSE}=\left(\frac{\sum_{i}^{N}\left(y_{i}-x_{i}\right)^{2}}{N}\right)^{1 / 2}
\end{gathered}
$$




$$
\operatorname{RMSE}(\%)=100 \frac{\left(\frac{\sum_{i}^{N}\left(y_{i}-x_{i}\right)^{2}}{N}\right)^{1 / 2}}{\bar{x}}
$$

Onde $y_{i}$ são os valores estimados, $x_{i}$ são os valores medidos, $N$ são o número de observações e $\bar{x}$ é o valor médio medido.

O indicativo MBE (Mean Bias Error) mostra o desvio das médias e fornece indicativos quanto o desempenho do modelo a longo prazo. Resultados positivos de MBE indicam que o modelo superestima os dados medidos e resultados negativos de MBE indicam que o modelo subestima os valores medidos. Quanto menor o valor absoluto de MBE, melhor o desempenho do modelo testado (Stone, 1993). A desvantagem deste indicativo é que uma superestimativa cancela uma subestimativa.

O RMSE (Root Mean Square Error) é a raiz quadrada do erro quadrático médio e informa quanto ao desempenho do modelo à curto prazo. Quanto menor seu valor, menor a dispersão dos dados em torno do modelo e melhor é a performance dos modelos. As desvantagens do RMSE são que alguns erros de grande proporção na soma podem causar acréscimos significativos nos valores de RMSE, e ele não diferencia superestimativa de subestimativa.

A realização de todos os cálculos e indicativos estatísticos foram através do software Origin.

\section{Resultados e Discussão}

\subsection{Análise dos modelos da literatura}

Os desempenhos dos modelos testados da literatura para aestimativa da irradiação solar global desenvolvidos em superfícies inclinadas foram confrontados com os dados medidos da irradiação solar em superfície vertical ao Norte. A figura 1 apresenta os gráficos da variação temporal dos valores estimados e medidos para cada modelo avaliado da literatura. 
Figura 1 - Variação temporal dos valores estimados e medidos para cada modelo avaliado da literatura.
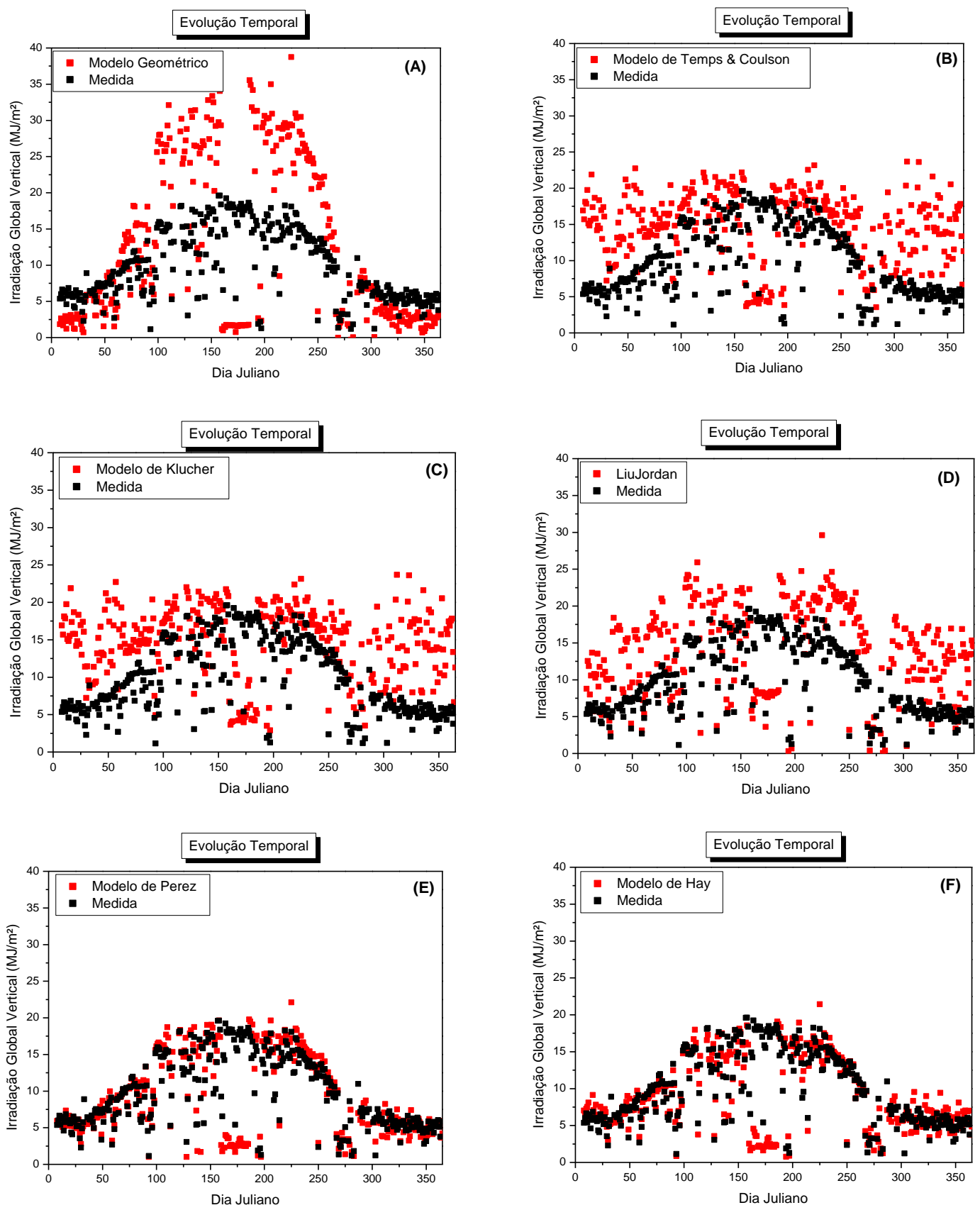

Fonte: Autores (2021).

Os resultados do desempenho do modelo Geométrico estão apontados na Figura 1-A. O modelo Geométrico apresentou o pior resultado na estimativa da irradiação solar em plano vertical no período do inverno, período em que a irradiação difusa é, em geral, anisotrópica, que não é levada em consideração pelo modelo. O modelo superestimou a irradiação global inclinada no período do inverno e subestimou nos meses de verão. Este modelo não considera as condições de céu, nem a porção da irradiação refletida, apenas utiliza o fator de correção geométrica de incidência dos raios solares na superfície inclinada $\left(R_{B}\right)$, o que justifica tal dispersão entre valores estimados e medidos.

Os resultados alcançados com o modelo de Temps e Coulson (1977) estão apontados na Figura 1-B. O modelo superestimou as medidas da irradiação solar global em superfície vertical ao longo do ano. Os valores que se aproximaram aos medidos foram durante o inverno, o que é esperado para este modelo, onde o termo $\left[1+\operatorname{sen}^{3}(\beta / 2)\right]$ auxilia razoavelmente bem 
para o brilho horizontal verificado em condições de céu claro, no qual a irradiação difusa próxima ao horizonte é mais alta do que no zênite do Sol (brilho horizontal).

A Figura 1-C ilustra o desempenho obtido com o modelo de Klucher (1979) para estimar a irradiação solar global que incidente em um sensor em uma superfície inclinada. No modelo de anisotrópico de Klucher (1979) observa-se, de modo geral, que o modelo superestima os níveis de irradiação solar global em todas as condições de céu, mudando de céu aberto à nublado, contudo, durante o inverno se aproximou dos valores medidos da irradiação global em superfície vertical, período em que a porção da irradiação solar difusa é predominantemente anisotrópica, com predomínio de dias de céu aberto. A função modeladora $\left[F=1-\left(H_{d}^{d} / H_{g}^{d}\right)^{2}\right]$ obteve melhores resultados em condições de céu claro, quando a razão da irradiação difusa pela global é pequena, onde $F$ tende a 1, o modelo aproxima-se do de Temps \& Coulson.

Os resultados obtidos com o modelo de Liu e Jordan (1973) estão indicados na Figura 1-D. O modelo de Liu e Jordan (1973), que é um modelo isotrópico, assume que a atividade da irradiação seja uniforme em todo hemisfério celeste para estimar a porção da irradiação difusa. O modelo superestimou as medidas durante o ano todo. Contudo, no mês de julho, subestimou as medidas da irradiação global em superfície vertical.

A Figura 1-E ilustra resultados da aplicação do modelo de Perez, et al. (1987) da estimativa a irradiação global incidente em uma superfície inclinada. Este modelo se ajustou bem às medidas da irradiação global em superfície vertical, o qual inclui as subcomponentes da irradiação difusa, isotrópica, brilho horizontal e circunsolar. A variação temporal acompanhou as medidas da irradiação solar global em superfície vertical tanto nos dias de céu nublado, quando as medidas apresentaram baixos níveis de irradiação global, até $10 \mathrm{MJ} / \mathrm{m}^{2}$, quanto para os dias de céu aberto, quando a irradiação global chega até $20 \mathrm{MJ} / \mathrm{m} / 2$.

A Figura 1-F mostra a variação anual dos valores estimados da irradiação solar global na superfície vertical alcançados com o modelo de estimativa de Hay (1979), que admite que a irradiação difusa composta das subcomponentes isotropicamente distribuídas vindas do hemisfério celeste e a circunsolar proveniente diretamente do disco solar. No verão, período que a irradiação difusa é predominantemente isotrópica, o modelo superestimou por volta de $3 \mathrm{MJ} / \mathrm{m}^{2}$ à mais que os dados medidos da irradiação global em superfície vertical. Desta forma, este modelo apresentou bons resultados na estimativa da irradiação solar global em superfície vertical, comparado aos valores medidos.

Visualmente, os modelos Geométrico, Klucher (1979), Temps e Coulson (1977) e Liu e Jordan (1963), apresentaram os piores desempenhos para as condições atmosféricas local enquanto os modelos de Perez, et al. (1987) e de Hay (1979) obtiveram os melhores desempenhos. Afim de quantificar esse desempenho à título de comparação, foram calculados os indicativos estatísticos MBE (MJ/m²), MBE(\%), RMSE (MJ/m²), RMSE (\%) e coeficiente angular obtidos na validação das equações de estimativa diária apresentados na Tabela 4. O melhor modelo de estimativa foi o de Hay, o qual forneceu menores valores nos indicativos estatísticos, MBE (\%) de 3,25\%, RMSE (\%) de 17,66\% e coeficiente angular de 1,01. Os piores desempenhos foram para os modelos de Klucher, Temps \& Clouson, Liu \& Jordan e Geométrico, onde os indicativos estatísticos mostraram que os modelos superestivaram os valores medidos, provavelmente porque esses modelos ou não contemplam a radiação refletida ou não contemplam os efeitos anisotrópicos da irradiação solar na fase de modelagem. 
Research, Society and Development, v. 10, n. 7, e38910716721, 2021

(CC BY 4.0) | ISSN 2525-3409 | DOI: http://dx.doi.org/10.33448/rsd-v10i7.16721

Tabela 4 - Indicativos estatísticos de desempenho dos modelos da literatura testados comparados com valores medidos.

\begin{tabular}{|c|c|c|c|c|c|}
\hline Modelo & $\begin{array}{c}\mathrm{MBE} \\
\left(\mathrm{MJ} / \mathrm{m}^{2}\right)\end{array}$ & $\begin{array}{c}\mathrm{MBE} \\
(\%)\end{array}$ & $\begin{array}{l}\text { RMSE } \\
\left(\mathrm{MJ} / \mathrm{m}^{2}\right)\end{array}$ & $\begin{array}{c}\text { RMSE } \\
(\%)\end{array}$ & $\begin{array}{c}\text { Coeficiente } \\
\text { angular }\end{array}$ \\
\hline Geométrico & 3,58 & 39,45 & 7,78 & 85,52 & 1,59 \\
\hline Klucher (1979) & 6,08 & 66,83 & 7,17 & 78,89 & 1,42 \\
\hline $\begin{array}{c}\text { Temps \& Coulson } \\
\text { (1977) }\end{array}$ & 5,96 & 63,53 & 7,04 & 74,97 & 1,43 \\
\hline $\begin{array}{l}\text { Liu \& Jordan } \\
\text { (1963) }\end{array}$ & 4,00 & 40,34 & 6,31 & 63,55 & 1,27 \\
\hline Perez (1987) & 0,42 & 4,64 & 1,72 & 18,91 & 1,05 \\
\hline Hay (1979) & 0,29 & 3,25 & 1,60 & 17,66 & 1,01 \\
\hline
\end{tabular}

Fonte: Autores (2021).

Para investigar o grau de ajustamento dos valores estimados à medida, foram construídos gráficos plotando o valor estimado no eixo Y e o valor medido no eixo X, sendo que os melhores desempenhos sejam alcançados para pontos coincidentes à reta ideal $\left(45^{\circ}\right)$. Nesse sentido, a figura 2 mostra a distribuição da irradiação solar global diária em plano vertical estimada através dos modelos da literatura testados em função dos valores medidos da irradiação solar global em superfície vertical. 
Figura 2 - Distribuição da irradiação solar global diária no plano vertical estimada através dos modelos Geométrico (A), Klucher (B), Temps e Coulson (C), Liu e Jordan (D), Perez (E) e Hay (F), em função dos valores medidos da irradiação solar global em superfície vertical.
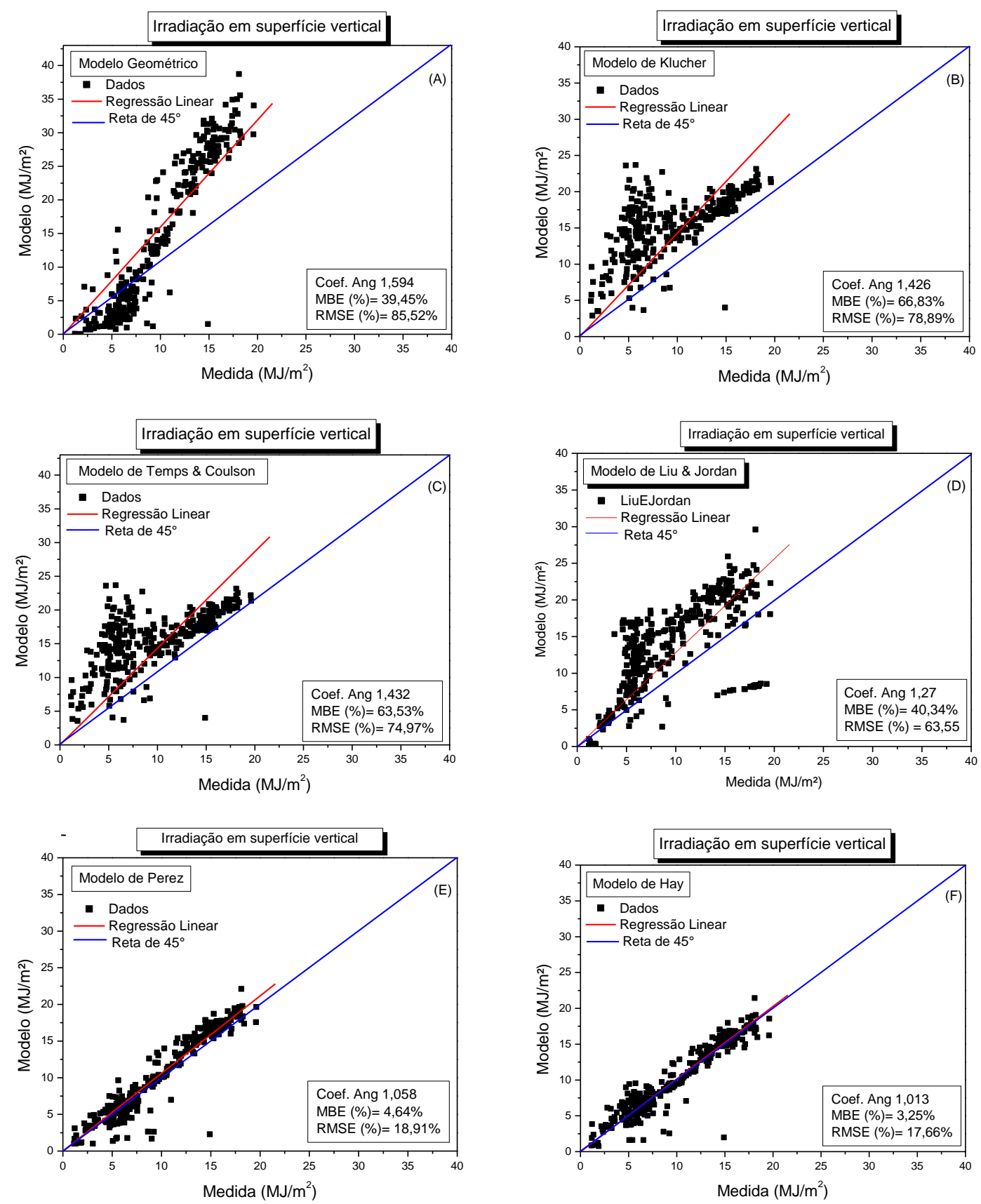

Fonte: Autores (2021).

A Figura 2-A mostra a validação do modelo Geométrico em função dos dados analisados da irradiação global em superfície vertical. Este modelo não se mostrou adequado, pois não considera o efeito da anisotropia da irradiação, fato que gerou grande espelhamento dos pontos $(\mathrm{RMSE}=85,52 \%)$ e superestimou os valores medidos, apresentando o indicativo MBE de $39,45 \%$ e e coeficiente angular de 1,59 .

A Figura 2-B mostra a validação do modelo anisotrópico de Klucher em função dos dados observados. Este modelo superestimou os dados observados, apresentando valores de $\mathrm{MBE}=66,83 \%$. O modelo também apresentou grande espalhamento dos pontos, gerando alto percentual do indicativo de dispersão (RMSE= 78,89\%) e coeficiente angular de 1,42. 
O modelo de Temps e Coulson apresentou os seguintes resultados nos indicativos estatísticos de desempenho: $\mathrm{MBE}=$ $63,53 \%$, RMSE $=74,97 \%$ e coeficiente angular de 1,43. O modelo superestimou os medidos, apresentou grande espalhamento e apresentou maior valor de coeficiente angular comparado com os outros modelos testados. Seu desempenho é mostrado na Figura 2-C.

A Figura 2-D apresenta a validação do modelo isotrópico de Liu e Jordan em função das medidas da irradiação global vertical. Este modelo apresentou altos valores nos indicativos estatísticos MBE e RMSE. O modelo superestimou os valores medidos, resultando em MBE $=40,34 \%$. O indicativo de espalhamento foi alto, sendo RMSE $=63,55 \%$, e coeficiente angular de 1,27 .

O desempenho da validação do modelo anisotrópico de Perez é mostrado na Figura 2-E. Comparado com os modelos anteriores, o modelo apresentou bom desempenho, com baixos valores de espalhamento e superestimativa. Os valores dos indicativos estatísticos são de MBE= 4,64\%, RMSE= 18,91\% e coeficiente angular de 1,05.

O modelo que apresentou o melhor desempenho foi o de Hay, o qual apresentou menores valores absolutos para todos os indicativos estatísticos, comparado com os outros modelos testados. Sendo MBE $(\%)=3,25 \%$, RMSE $(\%)=17,66 \%$ e coeficiente angular de 1,01. A validação do modelo de Hay é mostrada na Figura 2-F.

\subsection{Modelo proposto}

A irradiação solar global em superfície vertical é composta pelas parcelas direta, difusa e refletida, todas projetadas em superfície vertical, conforme já descrito na Equação 1. Dessa forma, o modelo de estimativa da radiação global em superfície inclinada foi desenvolvido em duas etapas. Na primeira etapa, as radiações direta e difusa em superfície horizontal foram projetadas para superfície vertical por meio da multiplicação do fator $\mathrm{Rb}$, se assemelhando ao modelo Geométrico. Na segunda etapa, a radiação refletida foi modelada através de regressão linear em função da irradiação solar global horizontal. A modelagem utilizou $75 \%$ dos dados e a validação os $25 \%$ restantes. A figura 3 mostra a correlação entre os dados de radiação refletida em função da radiação global horizontal com elevado coeficiente de determinação $\left(R^{2}=0,87\right)$, enquanto que a equação 11 já mostra o modelo com as três parcelas (direta, difusa e refletida) já incorporadas.

Figura 3 - Correlação entre os dados de radiação refletida em função da radiação global horizontal.

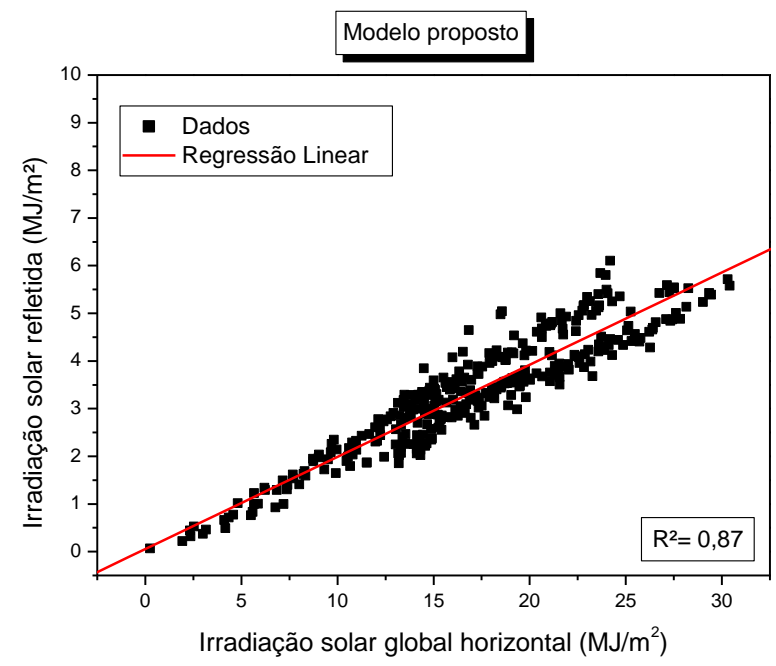

Fonte: Autores (2021).

$$
H_{\beta T}^{d}=H_{g}^{d} R_{B}+0,194 H_{g}^{d}+0,0531
$$


O desempenho do modelo proposto de estimativa da irradiação solar em plano vertical foi realizado por meio dos indicativos estatísticos MBE, RMSE e coeficiente angular. A Tabela 5 e a Figura 4 mostram a validação do modelo proposto.

Tabela 5 - Indicativos estatísticos de desempenho do modelo proposto comparado com valores medidos.

\begin{tabular}{c|c|c|c|c|c}
\hline Modelo & $\begin{array}{c}\text { MBE } \\
\left(\mathrm{MJ} / \mathrm{m}^{2}\right)\end{array}$ & $\begin{array}{c}\text { MBE } \\
(\%)\end{array}$ & $\begin{array}{c}\text { RMSE } \\
\left(\mathrm{MJ} / \mathrm{m}^{2}\right)\end{array}$ & $\begin{array}{c}\text { RMSE } \\
(\%)\end{array}$ & $\begin{array}{c}\text { Coeficiente } \\
\text { angular }\end{array}$ \\
\hline $\begin{array}{c}\text { Modelo } \\
\text { Proposto }\end{array}$ & 0,77 & 6,89 & 2,38 & 21,27 & 1,11 \\
\hline
\end{tabular}

Fonte: Autores (2021).

Figura 4 - Distribuição da irradiação solar global diária no plano vertical estimada através do modelo proposto em função dos valores medidos da irradiação solar global em superfície vertical.

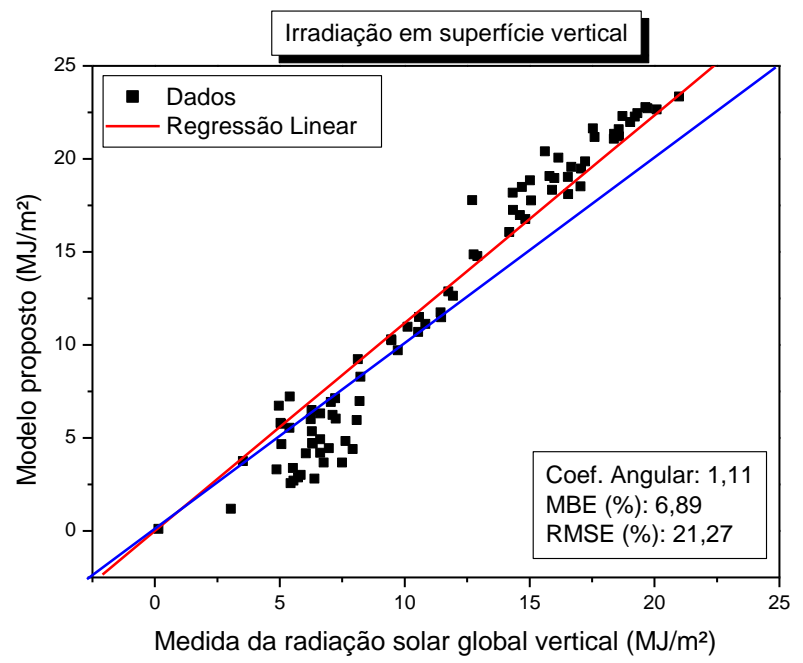

Fonte: Autores (2021).

O indicativo MBE resultou em 6,89\%, o que mostra que o modelo proposto superestimou as medidas da irradiação solar global vertical. Já no indicativo RMSE apresentou 21,27\% de espalhamento. O coeficiente angular foi de 1,11, porém, pela Figura 4, nota-se que, para os dados da radiação global vertical medidos inferiores à $10 \mathrm{MJ} / \mathrm{m} / 2$ o modelo apresentou estimativas abaixo da linha ideal, enquanto que para medidas superiores à $10 \mathrm{MJ} / \mathrm{m}^{2}$ o modelo apresentou estimativas acima da linha ideal.

O modelo proposto mostrou bons indicativos estatísticos, com desempenho superior em relação aos modelos de Liu e Jordan, Temps e Coulson, Klucher e Geométrico. No entanto, o modelo proposto foi inferior aos modelos de Hay e Perez, visto que estes dois últimos modelos tem uma parametrização mais complexa entre as parcelas da radiação com a geometria solar.

A fim de conferir o desempenho temporal do modelo proposto, a Figura 5 mostra a evolução temporal do modelo proposto comparado com os dados medidos da irradiação solar em superfície vertical. 
Figura 5 - Evolução temporal do modelo proposto comparado com os dados observados.

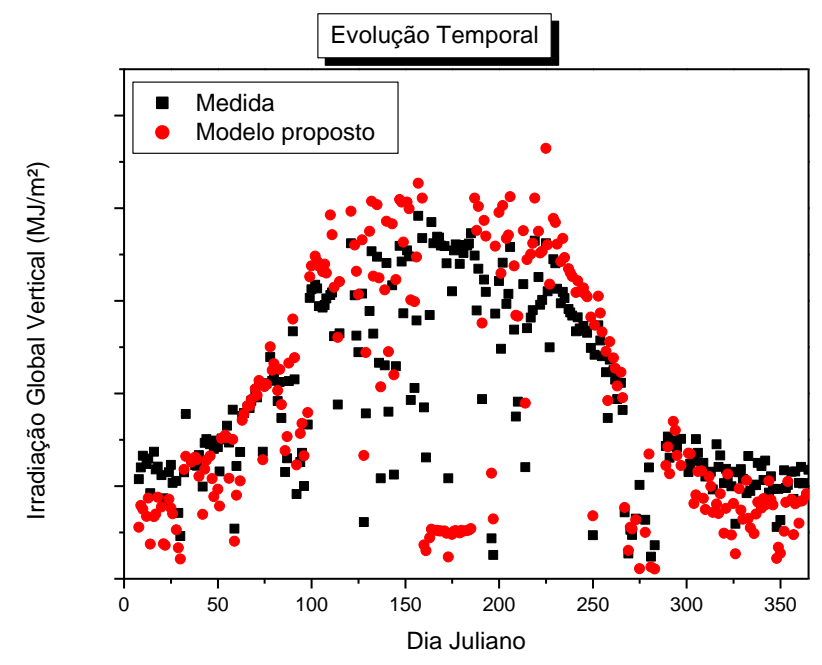

Fonte Autores.

Em geral, modelo apresentou bom desempenho ao longo do ano. Contudo, nos meses de verão houve subestimativa das medidas, enquanto que durante o inverno, houve superestimativa da medida.

\section{Conclusão}

Neste estudo, foi concluído que:

Avaliando o desempenho de cada modelo de estimativa da irradiação solar para superfícies inclinadas testados em superfície vertical, os que apresentaram o pior desempenho foram o Geométrico, Klucher, Temps e Coulson e de Liu e Jordan. Os modelos que apresentaram bons resultados comparados com as medidas foram os modelos de Hay e Perez, sendo o de Hay o modelo que mais se ajustou às medidas, e, portanto, o mais indicado para estimar a irradiação solar global em superfície vertical para Botucatu-SP;

$\checkmark \quad$ O modelo proposto mostrou bons indicativos estatísticos se comparados com os modelos da literatura;

$\checkmark \quad$ Mais estudos deverão ser realizados na tentativa de se melhorar a modelagem por meio da incorporação de efeitos anisotrópicos da radiação solar.

Para a continuidade de estudo, sugere-se analisar os dados das evoluções diurnas da radiação solar global em superfície vertical ao longo do ano, afim de se adquirir maiores conhecimentos da incidência da radiação solar global em superfície vertical horária para a região.

\section{Agradecimentos}

Conselho Nacional de Desenvolvimento Científico e Tecnológico - CNPq.

\section{Referências}

Barth, A., Wolfer, C. Lovato, A., \& Griebler, D. (2016). Avaliação da Irradiação Solar como Fonte de Energia Renovável no Noroeste do Estado do Rio Grande do Sul Através de Uma Rede Neural. Revista Eletrônica Argentina-Brasil De Tecnologias Da Informação E Da Comunicação, 1(5). 10.5281/zenodo.345585

Brito, M. C., Freitas, S., Guimaraes, S., Catita, C., \& Redweik, P. (2017). The importance offacades for the solar PV potential of a Mediterranean city using LiDAR data. Renewable Energy 111, 85-94.

Buriol, G. A., Estefanel, V., Heldwein, A. B., Prestes, S. D., \& Horn, J. F. C. (2012). Estimativa da radiação solar global a partir dos dados de insolação, para Santa Maria - RS. Ciência Rural, 42(9), 1563-1567. 
Research, Society and Development, v. 10, n. 7, e38910716721, 2021

(CC BY 4.0) | ISSN 2525-3409 | DOI: http://dx.doi.org/10.33448/rsd-v10i7.16721

Carvalho, C. C. S., Santos, T. C., Silva, G. C., Santos, L. V., Moreira, S. J. M., \& Botelho, L. F. R. (2014). Conforto térmico animal e humano em galpões de frangos de corte no semiárido mineiro. Revista Brasileira de Engenharia Agrícola e Ambiental, 18(7), 769-773.

Ceballos, J. C., \& Bottino, M. J. (2007). O modelo GL/CPTEC de radiação solar por satélite: potencial de informações para agrometeorologia. In: Anais XV Congresso Brasileiro de Agrometeorologia. Aracaju.

Codato, G, Oliveira, A, Soares, J, Escobedo, J, Gomes, E., \& Dal Pai A. (2007). Global and diffuse solar irradiances in urban and rural areas in southeast Brazil. Theoretical and Applied Climatology.93(1-2):57-73.

El-Maghlany, W. M., Teamah M. A., \& Tanaka H. (2015) Optimum design and orientation of the greenhouses for maximum capture of solar energy in North Tropical Region. Energy Conversion and Management, 105:1096-1104.

Hay, J. (1979) Calculation of monthly mean solar radiation for horizontal and inclined surfaces. Solar Energy. 23(4):301-307.

Iqbal, M. (1983). An Introduction To Solar Radiation. (1 ${ }^{\mathrm{a}}$ ed.): Academic Press. 390 p.

Klucher T. (1979) Evaluation of models to predict insolation on tilted surfaces. Solar Energy.23(2):111-114.

Liu, B., \& Jordan, R. (1963) The long-term average performance of flat-plate solar-energy collectors. Solar Energy.7(2):53-74.

Morettin, P. A., \& Toloi, C. M. C. (2004) Análise de Séries Temporais: Edgard Blücher.

Perez, R., Seals, R., Ineichen, P., Stewart, R., \& Menicucci D. (1987) A new simplified version of the perez diffuse irradiance model for tilted surfaces. Solar Energy. 39(3):221-231.

Redweik, P., Catita, C., \& Brito, M. (2013) Solar energy potential on roofs and facades in an urban landscape. Solar Energy.97:332-341.

Sevegnani, K. B., Ghelfi Filho, H., \& Silva, I. J. O. da. (1994). Comparação de vários materiais de cobertura através de índices de conforto térmico. Scientia Agricola, 51(1), 1-7.

Stanciu, C., Stanciu, D., \& Dobrovicescu, A. (2016) Effect of greenhouse orientation with respect to EW axis on its required heating and cooling loads, Energy Procedia, 85:498-504.

Stone, R. J. (1993). Improved statistical procedure for the evaluation of solar radiation estimation models. Solar Energy, 51(4), 289-91.

Subhashini, S., \& Thirumaran, K. (2018). A passive design solution to enhance thermal comfort in an educational building in the warm humid climatic zone of Madurai. Journal Of Building Engineering, 18, 395-407.

Varejão-Silva, M. A. (2006). Meteorologia e Climatologia: INMET. 463p.

Temps, R., \& Coulson, K. (1977) Solar radiation incident upon slopes of different orientations. Solar Energy.19(2):179-184.

Varejão Silva, M. A. (2006) Meteorologia e Climatologia: Versão Digital. 181 p.

Yao, R., Costanzo, V., Li, X., Zhang, Q., \& Li, B. (2018) The effect of passive measures on thermal comfort and energy conservation. A case study of the Hot Summer and Cold Winter climate in the Yangtze River region. Journal of Building Engineering, 15. pp. 298-310. ISSN 2352-7102

Yildırım, H, Teke, A. \& Antonanzas-Torres, F. (2018) Evaluation of classical parametric models for estimating solar radiation in the Eastern Mediterranean region of Turkey. Renewable and Sustainable Energy Reviews.82:2.053-2.065. 